\title{
Contrast Limited Adaptive Histogram Equalization for Qualitative Enhancement of Myocardial Perfusion Images
}

\author{
Neethu M. Sasi, V. K. Jayasree \\ Govt. Model Engineering College, Cochin University of Science and Technology, Thrikkakkara, Kerala, India \\ Email: neethumsasi@gmail.com, jayasreevk@gmail.com \\ Received May 2013
}

\begin{abstract}
This paper establishes an efficient color space for the contrast enhancement of myocardial perfusion images. The effects of histogram equalization and contrast limited adaptive histogram equalization are investigated and the one which gives good enhancement results is extended to the suitable color space. The color space which gives better results is chosen experimentally. Uniqueness of this work is that contrast limited adaptive histogram equalization technique is applied to the chrominance channels of the cardiac nuclear image, leaving the luminance channel unaffected which results in an enhanced image output in color space.
\end{abstract}

Keywords: Myocardial Perfusion Images; Single Photon Emission Computed Tomography; Histogram Equalization

\section{Introduction}

Medical imaging applies a lot of digital image processing techniques for better interpretation. Different enhancement techniques are available in literature for improving the quality of medical images. The major challenge in this area is that a specific algorithm which gives better results for a particular type of application may fail in giving good results for another type of application. Different popular imaging modalities are now available for detecting cardiac disorders. This work mainly concentrates on color images obtained from Single Photon Emission Computed Tomography (SPECT) systems which are designed to analyze the functioning of the heart [1]. This work is meant for improving the pictorial representation of the above mentioned images, thus providing a more accurate diagnosis of cardiac abnormalities.

One of the many purposes of taking nuclear heart scan is to check the blood flow to the heart muscle. If the heart muscle is not getting enough blood it may be a sign of coronary heart disease. When a nuclear heart scan is done for this purpose, it is called myocardial perfusion scanning [2].

Different image enhancement techniques are available in the literature [3]. Primarily, an image enhancement technique is done to process an image so that the resulting image gives more visual information than the original image. Nuclear medicine images suffer from a large amount of blur. A few studies are available in literature regarding the enhancement of nuclear images. A method of enhancement of noisy planar nuclear images using mean field annealing was proposed by Falk et al. [4]. Wang et al. uses a combined technique of mean field annealing and gradient edge detection to extract the boundary of left ventricle in [5]. The work discussed so far focused on gray scale images.

Contrast limited adaptive histogram equalization have been successfully proven to be effective in biomedical image analysis. Pisano et al. proposed contrast limited adaptive histogram equalization for detecting abnormalities in dense mammograms in [6].

This work presents the effect of contrast limited adaptive histogram equalization techniques on myocardial perfusion images in color space. The paper is organized as follows. The basic histogram equalization technique is presented and it discusses about contrast limited adaptive histogram equalization. The method suitable for myocardial images is explained and the experimental results are analyzed and finally the paper is concluded.

\section{Histogram Equalization}

Histogram equalization is one of the well-known enhancement techniques. In histogram equalization [3], the dynamic range and contrast of an image is modified by altering the image such that its intensity histogram has a desired shape. This is achieved by using cumulative distribution function as the mapping function. The intensity levels are changed such that the peaks of the histogram are stretched and the troughs are compressed. If a digital image has $N$ pixels distributed in $L$ discrete intensity levels and $n_{k}$ is the number of pixels with intensity level $i_{k}$ 
and then the probability density function (PDF) of the image is given by Equation (1). The cumulative density function (CDF) is defined in Equation (2).

$$
\begin{aligned}
& f_{i}\left(i_{k}\right)=\frac{n_{k}}{N} \\
& F_{k}\left(i_{k}\right)=\sum_{j=0}^{k} f_{i}\left(i_{j}\right)
\end{aligned}
$$

Though this method is simple, it fails in myocardial nuclear images since the gray values are physically far apart from each other in the image. Due to this reason, histogram equalization gives very poor result for myocardial images.

\section{Contrast Limited Adaptive Histogram Equalization}

In contrast limited histogram equalization (CLHE), the histogram is cut at some threshold and then equalization is applied. Contrast limited adaptive histogram equalization (CLAHE) is an adaptive contrast histogram equalization method [7-10], where the contrast of an image is enhanced by applying CLHE on small data regions called tiles rather than the entire image. The resulting neighboring tiles are then stitched back seamlessly using bilinear interpolation. The contrast in the homogeneous region can be limited so that noise amplification can be avoided.

\section{Contrast Limited Adaptive Histogram Equalization for Myocardial Perfusion Images in Color Space}

In this method, the image which is read in RGB space is converted into the color space with a luminance $(Y)$ and two chrominance components $(\mathrm{Cb}, \mathrm{Cr})$ by using the relation given in Equation (3).

$$
\left[\begin{array}{c}
Y \\
C b \\
C r
\end{array}\right]=\left[\begin{array}{c}
16 \\
128 \\
128
\end{array}\right]+\left[\begin{array}{ccc}
65.481 & 128.553 & 24.966 \\
-37.797 & -74.203 & 112.000 \\
112.000 & -93.786 & -18.214
\end{array}\right]\left[\begin{array}{l}
R \\
G \\
B
\end{array}\right]
$$

The two chrominance channels are separated and for each chrominance channel the number of rectangular contextual tiles into which the image is divided is obtained. The optimal value for this is decided experimentally. Uniform distribution is used as the basis for creating the contrast transform function. Let $i_{c_{-} \min }$ and $i_{c_{-} \max }$ be the minimum and maximum permissible intensity levels and the optimal value of this clip limit is also set. Let $F_{k}\left(i_{c_{-} \text {in }}\right)$ be the cumulative distribution function for input contextual tile $i_{c_{-} \text {in }}$. Then the expression of the modified chrominance channel tile with uniform distribution is given in Equation (4). The flowchart for the method is given in Figure 1.

$$
i_{c_{-} \text {out }}=\left[i_{c_{-} \max }-i_{c_{-} \min }\right] * F_{k}\left(i_{c_{-} \text {in }}\right)+i_{c_{-} \min }
$$

\section{Experimental Results}

The algorithm described in Section 4 has been applied to myocardial perfusion images obtained from a SPECT device. Nuclear image data sets from ten different patients, including both normal and abnormal, have been tested with the proposed algorithm in the follow up experiments. This paper has included a few images and their results following the enhancements. The original myocardial image is given in Figure 2. The histogram of the image can be seen in Figure 3. The equalized histogram by using simple histogram equalization is given in Fig-

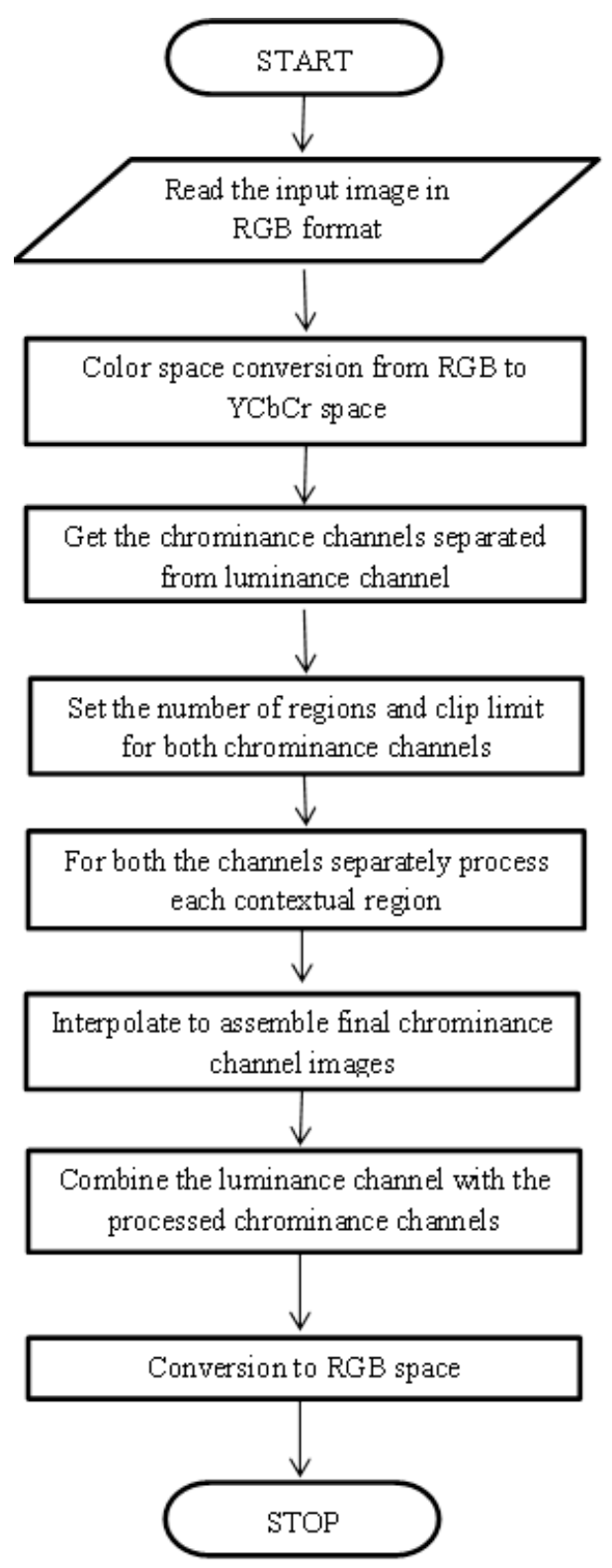

Figure 1. Enhancement method for cardiac SPECT image. 


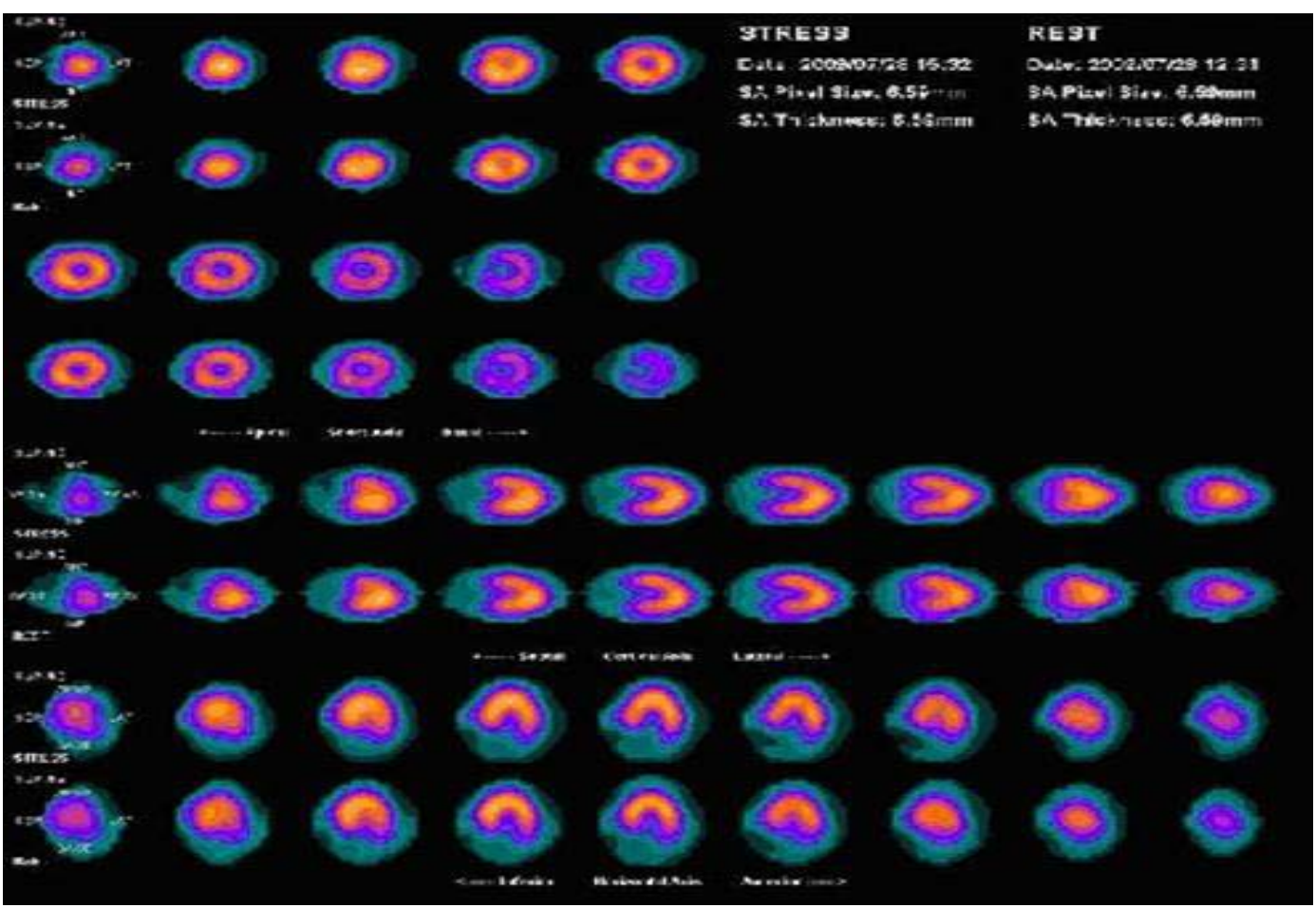

Figure 2. Original cardiac SPECT image.

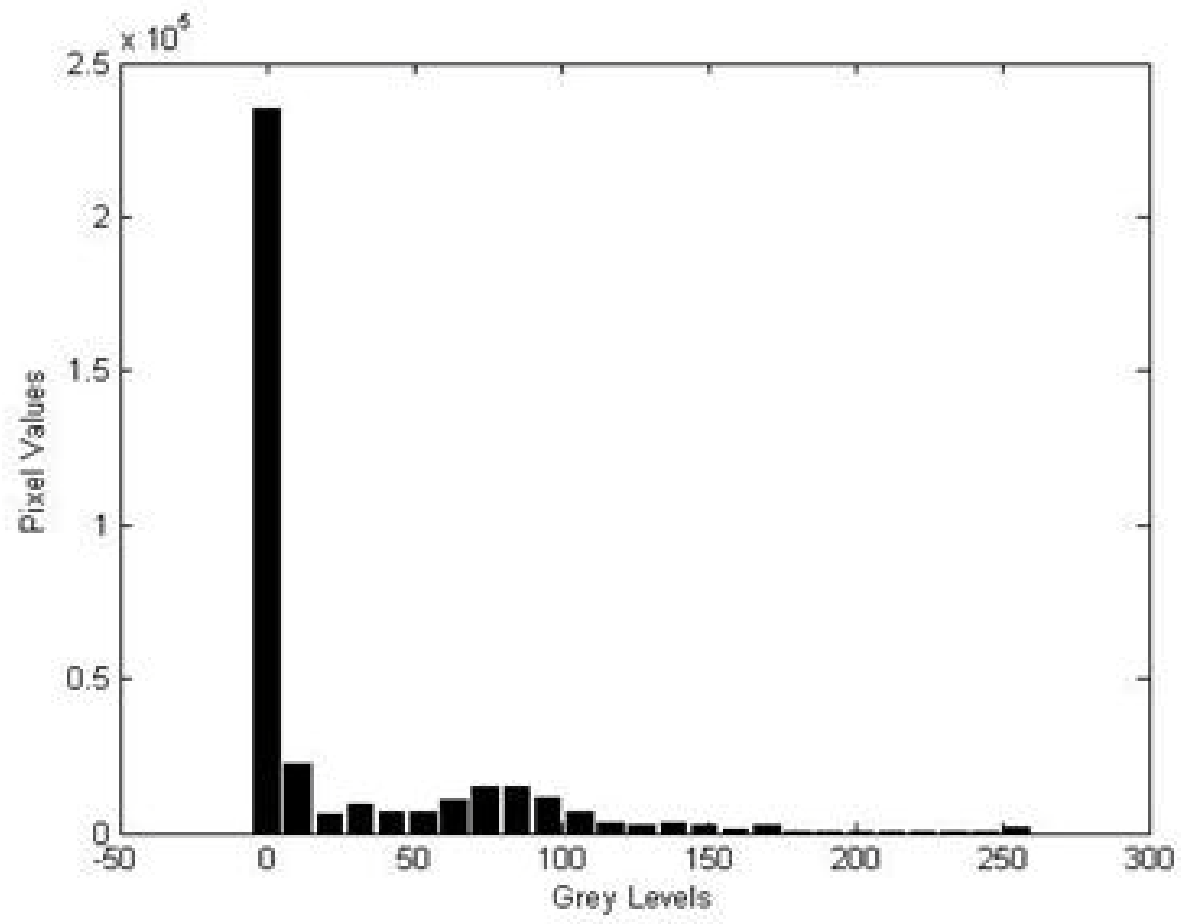

Figure 3. Histogram of the image shown in Figure 2.

ure 4 and the correspondingly processed image is shown in Figure 5. The loss of information due to over enhancement is evident if we compare the original image in Figure 2 and the enhanced image in Figure 5.
The proposed method extracts the chrominance channels from the original image. Then each channel is divided into rectangular tiles of size $32 \times 32$ and the clip limit is set at 0.01 . The above parameters have been cho- 


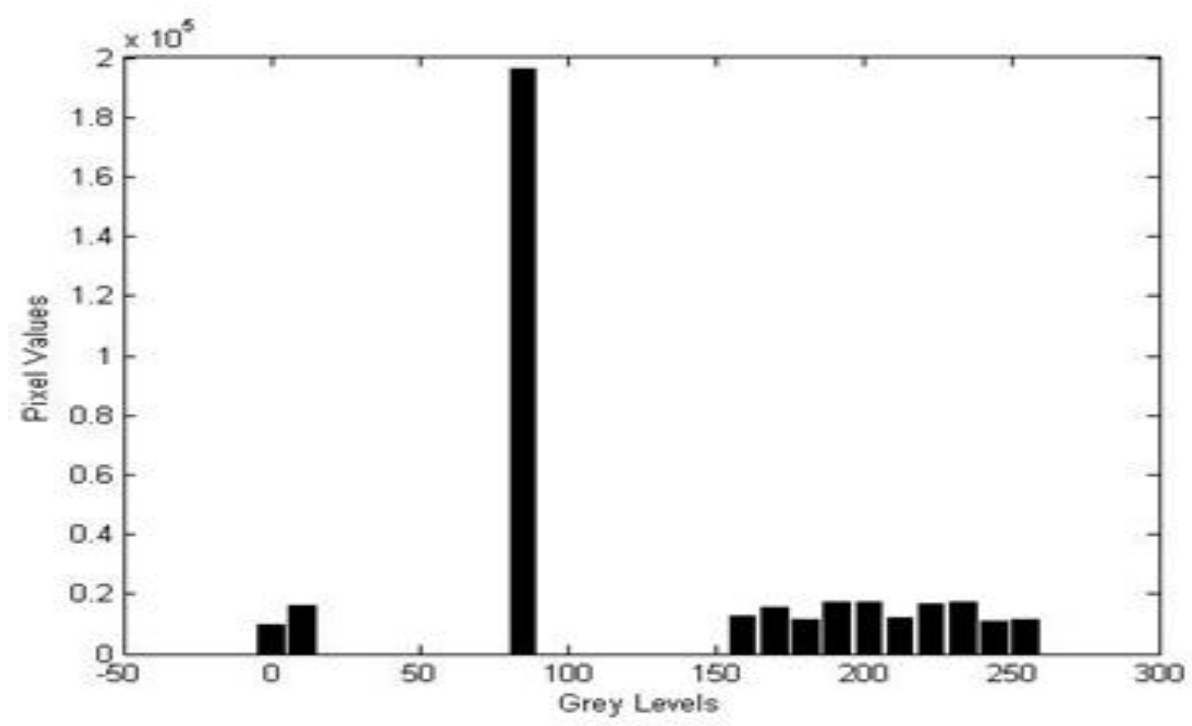

Figure 4. Equalized histogram.

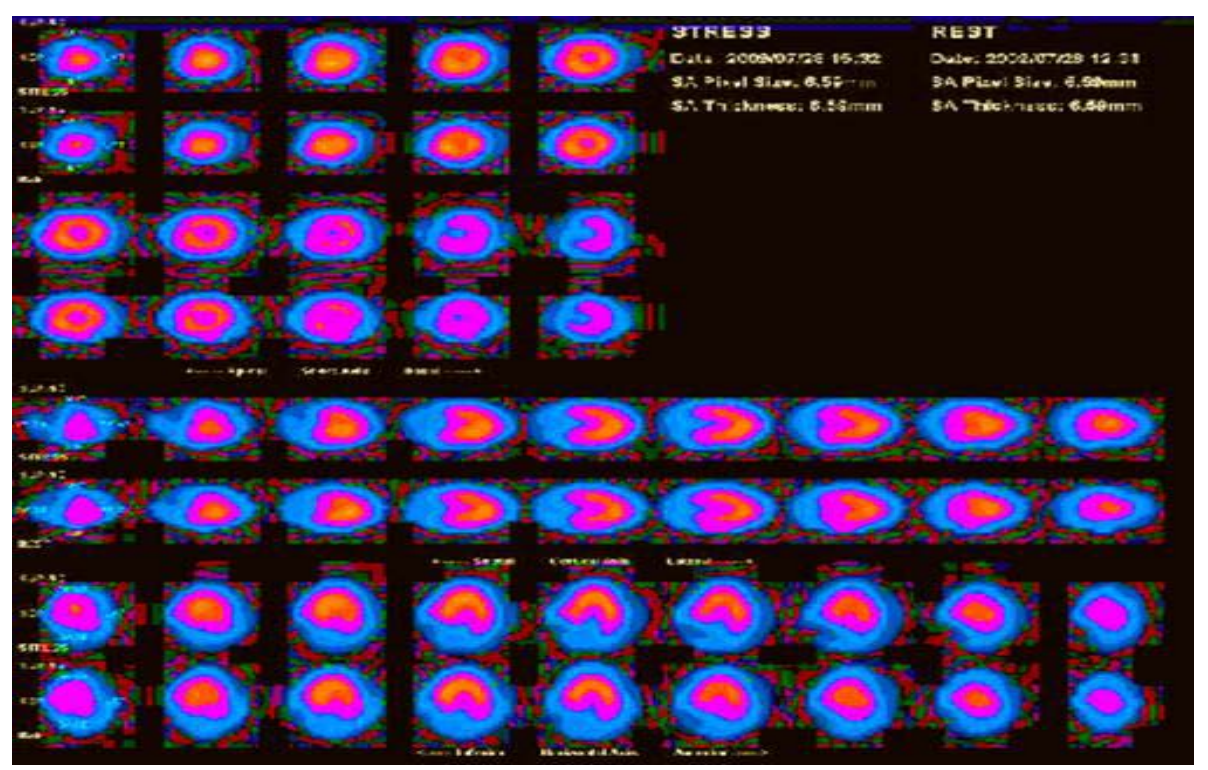

Figure 5. Image after simple histogram equalization.

sen by conducting experiments using different image datasets of the same type. Better histogram equalization is obtained by the method described in Section 4 using the said parameters. The contrast of the image has registered an improvement without loss in information as seen in the result in Figure 6. The histogram of the enhanced image by the proposed method is given in Figure 7 . Comparing Figure 2, Figure 5 and Figure 6, it can be concluded that the proposed method provides better enhancement conserving image data integrity.

Figure 8 shows a stress-rest portion of the SPECT image. The result of simple histogram equalization is given in Figure 9. The result of applying proposed extension of CLAHE is given in Figure 10. The result in Figure 10 concludes that the extended method of CLAHE gives better enhancement for cardiac SPECT images in color space.

\section{Conclusion}

The paper investigates the effect of histogram enhancement techniques on myocardial perfusion images from SPECT scan devices. Due to wide difference in intensity values, simple histogram equalization fails in these types of images as shown in Figure 5 and Figure 9. The simple histogram method suffers from intensity saturation which results in information loss which is not acceptable in the case of medical images. Visually appreciable re- 


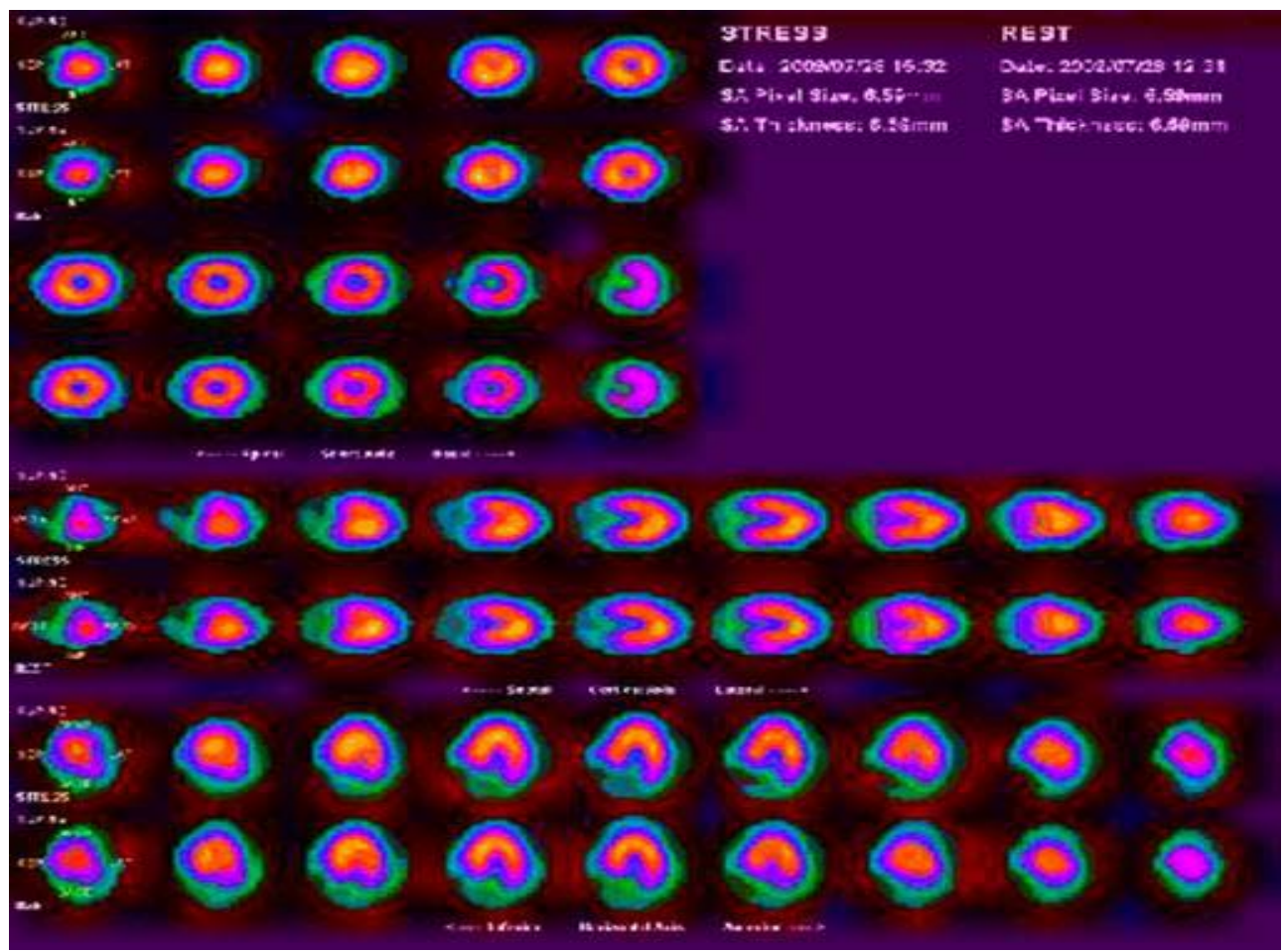

Figure 6. Processed image using proposed algorithm.

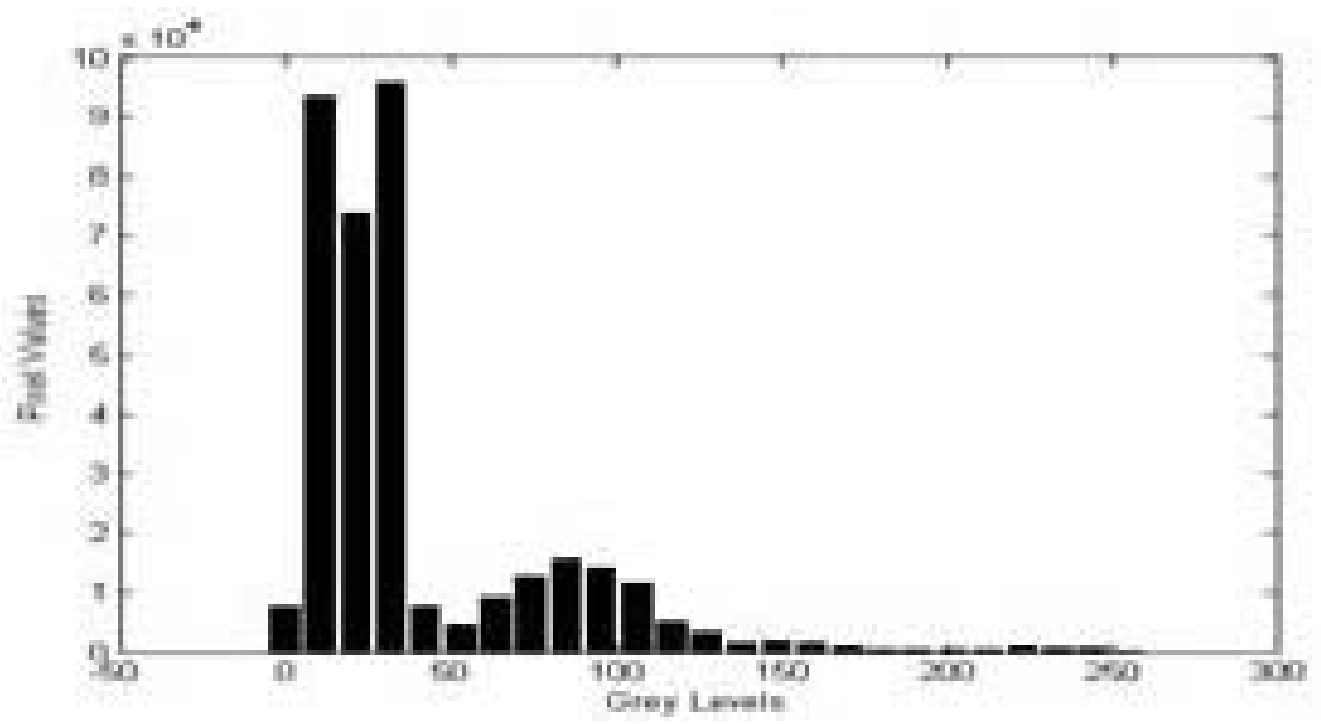

Figure 7. Histogram of the image shown in Figure 6.

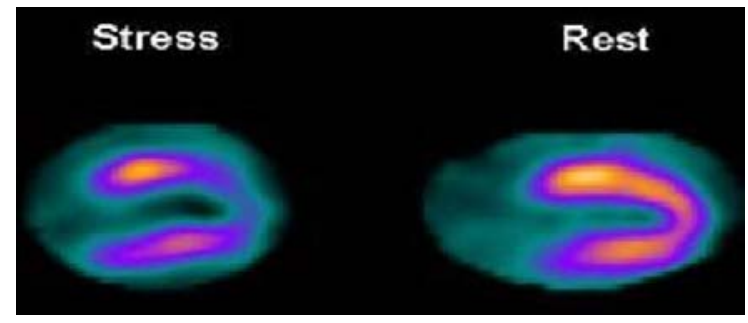

Figure 8. A portion of SPECT image showing stress and rest conditions of the heart.

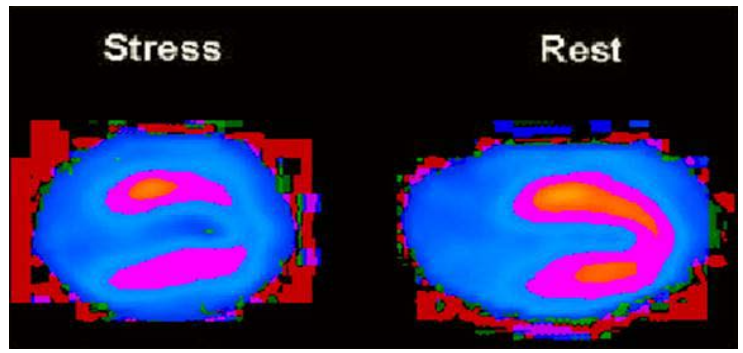

Figure 9. Image after simple histogram equalization. 


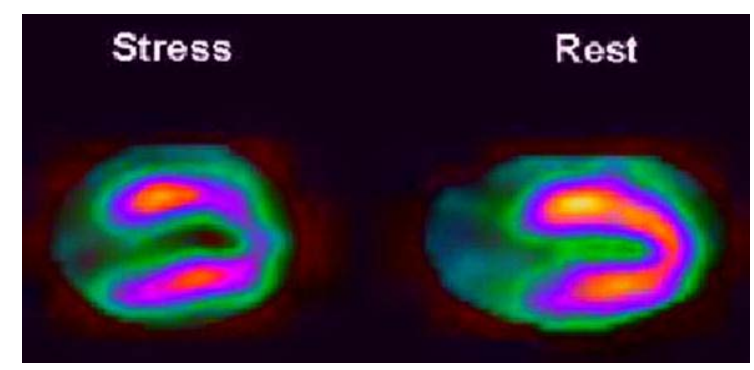

Figure 10. Processed image through proposed algorithm.

sults with image data integrity are obtained by extending CLAHE method to suitable color space containing chrominance and luminance components. This technique effectively improves the visual interpretation of these types of images thereby ensuring a more accurate guidance to the post-diagnostic procedures in cardiac ailments.

\section{REFERENCES}

[1] M. T. Madsen, "Recent Advances in Spect Imaging," The Journal of Nuclear Medicine, Vol. 48, No. 4, 2007, pp. 661-673. http://dx.doi.org/10.2967/jnumed.106.032680

[2] S. Burrell and A. MacDonald, "Artifacts And Pitfalls in Myocardial Perfusion Imaging," Journal of Nuclear Medicine Technology, Vol. 34, No. 4, 2006, pp. 193-211.

[3] R. C. Gonzalez, R. E. Woods and S. L. Eddins, "Digital Image Processing,” 2nd Edition, Pearson Education, New Jersey, 2002.

[4] D.L. Falk, D. M. Rubin and T. Marwala, "Enhancement of Noisy Planar Nuclear Medicine Images Using Mean
Field Annealing," World Congress on Medical Physics and Biomedical Engineering, IFMBE Proceedings, Vol. 4, Track 14, Korea, 2006, pp. 2415-2418.

[5] C. X. Wang, L. Small, W. E. Snyder and R. Williams, "Edge Detection in Gated Cardiac Nuclear Medicine Images," IEEE 7th Symposium on Computer Based Medical Systems, Winston-Salem, 10-12 June 1994, pp. 28-33.

[6] E. D. Pisano, S. Zong, B. M. Hemminger, M. DeLuca, R. E. Johnston, K. Muller, M. P. Braeuning and S. M. Pizer, "Contrast Limited Adaptive Histogram Equalization Image Processing to Improve the Detection of Simulated Spiculations in Dense Mammograms," Journal of Digit Imaging, Vol. 11, No. 4, 1998, pp. 193-200. http://dx.doi.org/10.1007/BF03178082

[7] R. Garg, B. Mittal and S. Garg, "Histogram Equalization Techniques for Image Enhancement,” International Journal of Electronics and Communication Technology, Vol. 2, No. 1, 2011, pp. 107-111.

[8] S. Srinivasan and N. Balram, "Adaptive Contrast Enhancement Using Local Region Stretching,” Proceedings of ASID ’06, New Delhi, 8-12 October 2006, pp. 152-155.

[9] S. K. Shome and S. R. K. Vadali, "Enhancement of Diabetic Retinopathy Imagery Using Contrast Limited Adaptive Histogram Equalization," International Journal of Computer Science and Information Technologies, Vol. 2, No. 6, 2011, pp. 2694-2699.

[10] J. A Stark, “Adaptive Image Contarst Enhancement Using Generalizations of Histogram Equalization,” IEEE Transactions on Image Processing, Vol. 9, No. 5, 2000, pp. 889-894. http://dx.doi.org/10.1109/83.841534 